\title{
Application of Viscoelastic Materials in Ship Collision
}

\author{
Haonan Gong* \\ BASIS International School Guangzhou, Guangzhou, Guangdong Province, China \\ *Corresponding author: Haonan Gong, andygonghaonan@ hotmail.com
}

\begin{abstract}
With the expansion of global trade routes, ship collision has become a major problem. This article proposes an approach by laying viscoelastic material on the inner side of the ship to increase the crashworthiness. By using the nonlinear finite element model software, this study simulates ship collisions as well as models and analyzes ships along with viscoelastic materials. The results from the simulation suggest that viscoelastic materials can effectively protect ships during collisions.
\end{abstract}

Keywords: Viscoelastic materials; Crashworthiness; Ship collision

Publication date: November 2021; Online publication: November 30, 2021

\section{Introduction}

With the development and globalization, sea transportation has gradually become a major route for trade. However, the risk of ship collisions has also increased. The damage of hulls caused by ship collisions results in the loss of life and property. In order to prevent the occurrence of such accidents, the commonly used measures today can be divided into active and passive protective measures. Active protective measures refer to the early warning of risks in order to avoid accidents. Passive protective measures refer to enhancing a ship's crashworthiness. Due to its large area, the sides of a ship are common parts to be damaged by collisions. On account of this reason, much research has been done in seeking an effective approach to improve the crashworthiness of the sides. Kitamura proposed two novel side structures for ships: frame panel and corrugated panel ${ }^{[1]}$. He made a comparison between them. The experimental results demonstrated that the capacity of frame panel to resist impact is obviously better than that of corrugated panel under a similar impact force. Lee and other researchers proposed that the structure of NOAHS II could absorb the impact energy to a large extent by replacing the plates in the double-layer structure ${ }^{[2]}$. Based on the aforementioned research, Wang and $\mathrm{Gu}$ put forward the CCT (circular tubes) structure ${ }^{[3]}$, which is composed of I-frame and thin-walled circular tubes. After using the finite element method to simulate and calculate, Wang concluded that the CCT structure is significantly superior to the former structure in terms of impact resistance. However, this structure increases the weight of ships along with a relatively complicated manufacturing process; thus, it is not practical to the application on civil transport ships. Ludolphy proposed a new type of double-deck structure, which involves the application of internal Yshaped stiffeners to the sideboards of ships ${ }^{[4]}$. In subsequent studies, Klanac proved that it has better crashworthiness than other structures by comparing traditional double-layer structures and other structures with this Y-shaped structure ${ }^{[5]}$. As the rigidity of the Y-shaped stiffeners is lower, it alleviates the energy due to the impact more effectively. In a study conducted by Crupi ${ }^{[6]}$, he proposed two aluminum sandwich materials, AFS (aluminum foam sandwich) and honeycomb panels, which have the advantages of being lighter in weight and stronger buffering performance compared to traditional steel structures. Through actual experiments, the study revealed that this energy dissipation material can effectively reduce the stress 
and deformation of a ship when it receives an impact.

Instead of improving the structural design of ships, this article introduces a different approach of passive protection during ship collision by applying another material on the sides of ships. Due to their special properties, viscoelastic materials can produce relatively hysteretic strain and stress when they undertake load, which can help ships to buffer the impact brought by collision. This study uses the finite element method to determine the crashworthiness of this material and discuss its application on the sides of ships.

\section{Principle}

Viscoelastic materials are rubbery at room temperature and have the characteristic of large damping. Its weight is also lighter than that of traditional materials. In terms of microscopic observation, when these materials receive an external force, their molecular chains would entangle, resulting in the loss of force. Due to the special molecular structure of the material, viscoelastic materials have two characteristics: elasticity and viscosity. Viscoelastic materials would deform when the force magnitude received changes, absorbing the energy of the impact as a cushioning. When the external force disappears, they would rebound to the initial state. Although they cannot be completely restored to their original state, these materials would release the absorbed impact energy in the process of recovery. This is known as the elastic property. In regard to the property of viscosity, when there is impact to the structure, the response of the strain would delay after the change of stress. In addition, there would be certain plastic deformation after being impacted. In other words, the increase in stress in the early stage of collision does not produce a large strain, and the stress caused by the strain would increase slowly in the later stage. Due to this special characteristic, strain $\gamma$ and stress $\tau$ would form a hysteresis loop as shown in Figure $1^{[7]}$.

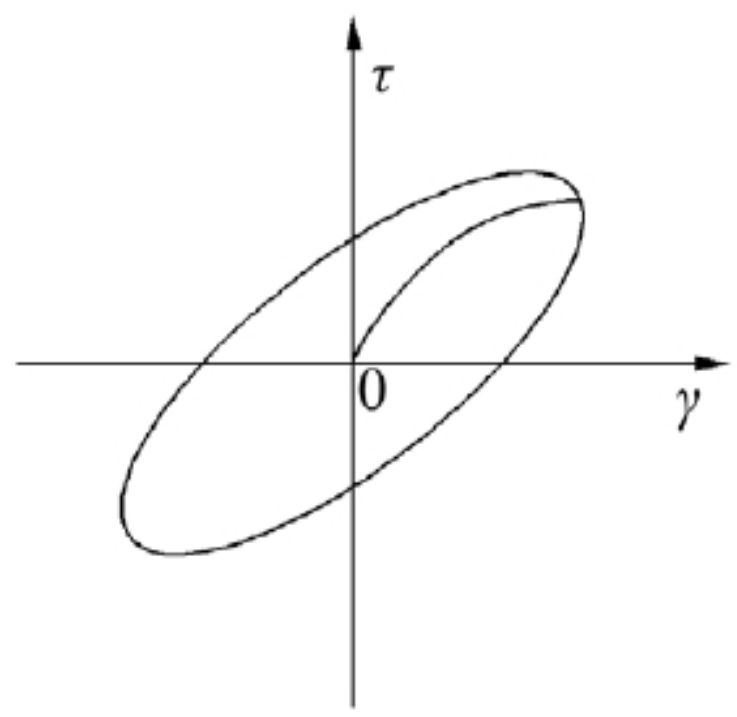

Figure 1. Stress-strain hysteresis loop of viscoelastic materials

The special properties of this hysteresis loop can increase the energy dissipation capacity of viscoelastic materials as a buffering material; that is, when a collision occurs, the growth rate of the stress subjected to the viscoelastic material would gradually decrease with the increase of strain. By applying this to ship collisions, the impact energy brought by collisions can be reduced effectively, thus reducing the damage to ships. 


\section{Simulation of laying viscoelastic materials on ships}

Using Abaqus, a finite element analysis software, this study models a simplified 87,000 tons cargo ship ${ }^{[8]}$. This model selects part of the ship's parallel middle body but overlooks the longitudinal bulkhead and stiffeners in each longitudinal direction. The visualized model of this section is shown in Figure 2, its finite element model is shown in Figure 3, and the model with viscoelastic materials added to the inner side of the ship is shown in Figure 4.

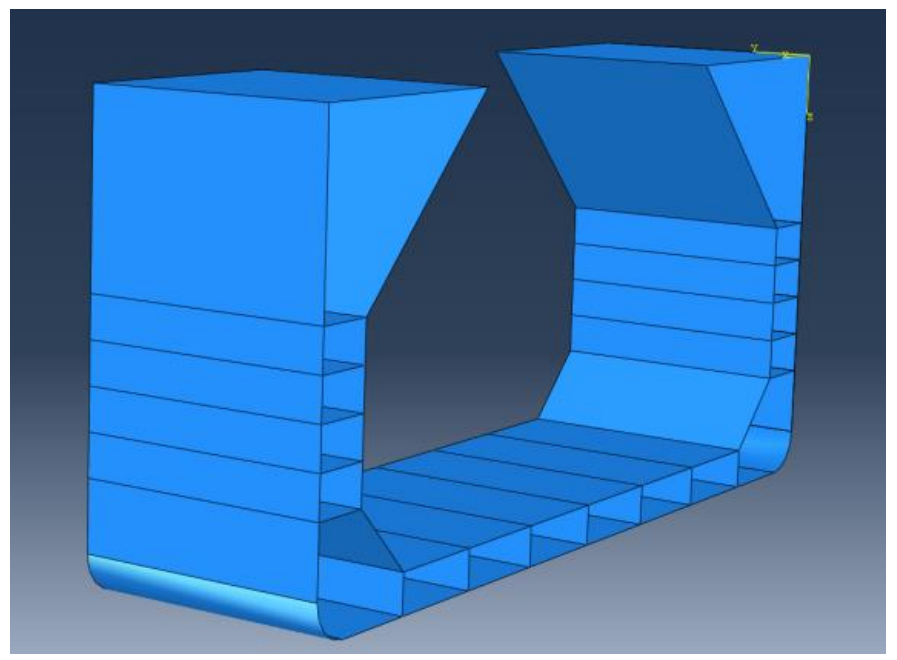

Figure 2. Ship's geometry model

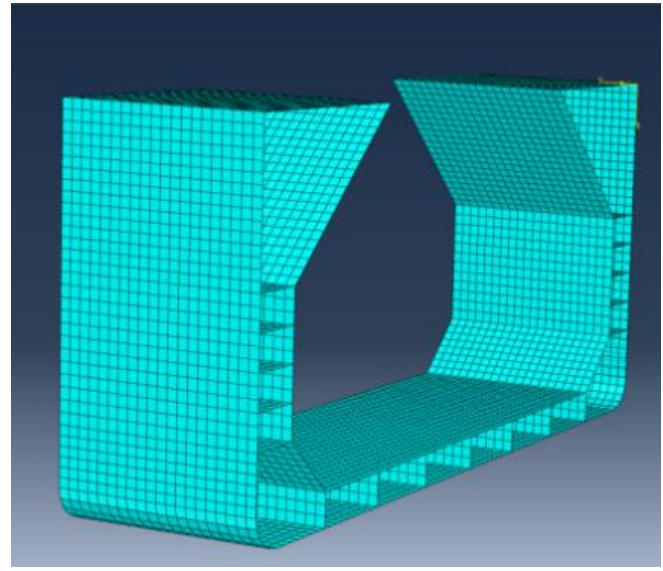

Figure 3. Ship's finite element model

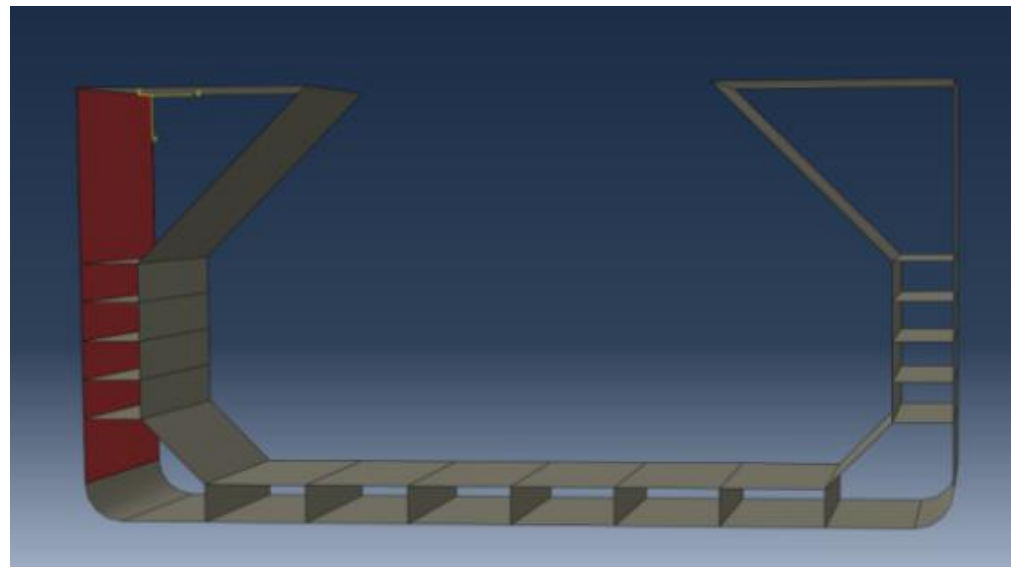

Figure 4. Viscoelastic materials laid over the ship (red parts)

It should be noted that this study primarily focuses on the protective effect of viscoelastic materials on the hull in a collision, so viscoelastic materials are only laid on one side of the ship.

In this study, the impact from collisions on the simulated ships is analyzed by the finite element method. The simulations are carried out before and after laying viscoelastic materials, respectively. The boundary conditions of the collision are set as shown in Figure 5.

The impact material is a cube of 1 meter in length, width, and height. In order to simulate the effect of gravity, this cube is accelerated at $9.8 \mathrm{~m} / \mathrm{s}^{2}$ and smashed vertically into the hull.

The cube is made up of steel, of which the parameters of its model are shown in Table 1. The ship uses the same steel material. Table 2 shows the parameters of the model with viscoelastic materials applied on the ship's side. 


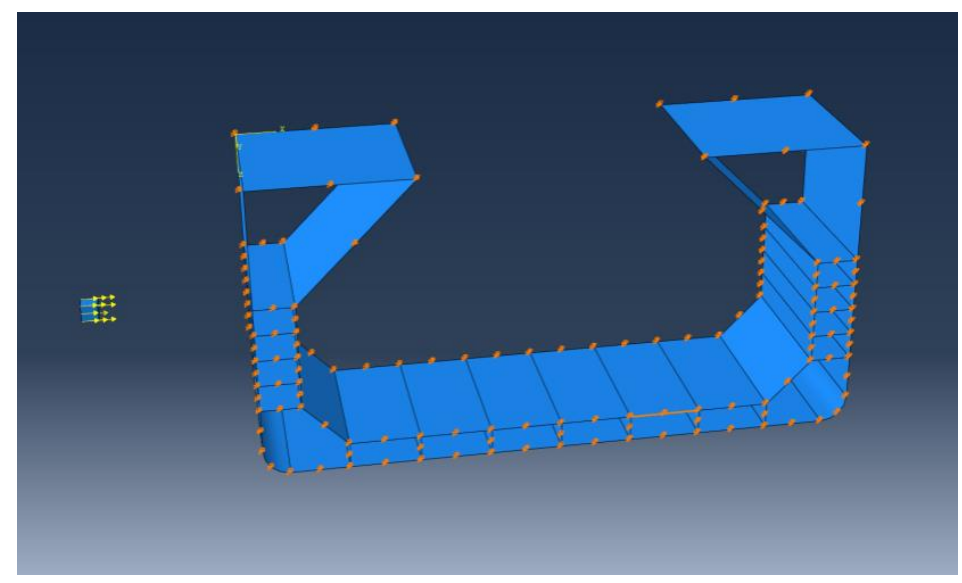

Figure 5. General view of collision

Table 1. Model parameters of steel materials

\begin{tabular}{ccc}
\hline Density $/ \mathrm{km} \cdot \mathrm{m}^{-3}$ & Young's modulus $/ \mathrm{N} \cdot \mathrm{m}^{-2}$ & Poisson's ratio \\
\hline $7.8 \times 10^{2}$ & $2.1 \times 10^{11}$ & 0.3 \\
\hline
\end{tabular}

Table 2. Model parameters of viscoelastic materials

\begin{tabular}{ccc}
\hline Density $/ \mathrm{km} \cdot \mathrm{m}^{-3}$ & Young's modulus $/ \mathrm{N} \cdot \mathrm{m}^{-2}$ & Poisson's ratio \\
\hline $9.681 \times 10^{2}$ & $1.794 \times 10^{6}$ & 0.3 \\
\hline
\end{tabular}

In the calculation of viscoelastic materials, the complex constant viscoelastic model is the most commonly used model in engineering calculation because of its characteristics of describing the simple harmonic motion of viscoelastic materials and linearizing the solution of complex viscoelastic materials mathematical model.

$$
\mathrm{E}_{\mathrm{v}}=\mathrm{E}_{0}\left(1+\mathrm{i} \eta_{\mathrm{v}}\right)
$$

In this formula, $E_{0}$ is the energy stored in the viscoelastic material, and $\eta_{\mathrm{v}}$ is the dissipation factor of that material.

This study compares the results of the two collision simulations and analyzes the crashworthiness of viscoelastic materials.

\subsection{Displacement comparison}

During the whole collision process, the displacement curve of the collision contact point changing with time is shown in Figure 6.

First, the displacement amplitude generated by collision on ships equipped with viscoelastic materials is smaller than that on ships without such materials. Hence, viscoelastic materials are able to greatly help ships reduce irreparable damages caused by the large amplitude of displacements.

Second, the elastic property of viscoelastic materials allows ships to bounce back more quickly with less shock. As shown in Figure 6, the slope of the ship with viscoelastic materials is larger after the collision. In subsequent waves, the viscoelastic ship shows a smaller slope and amplitude of displacement change. These illustrations demonstrate the capacity of viscoelastic materials in aiding the damaged part of the ship to quickly return to its original displacement. This graph also proves that viscoelastic materials can absorb 
the energy generated by the impact and release it to resist subsequent waves. This is the "elastic" property of viscoelastic materials.

In addition, compared to ships without viscoelastic materials, this material ensures that ships do not undergo a second wave immediately after the first return to the initial displacement but instead, remain in that shape for a period of time, as can be seen in the graph. It successfully reflects the hysteresis of viscoelastic materials.

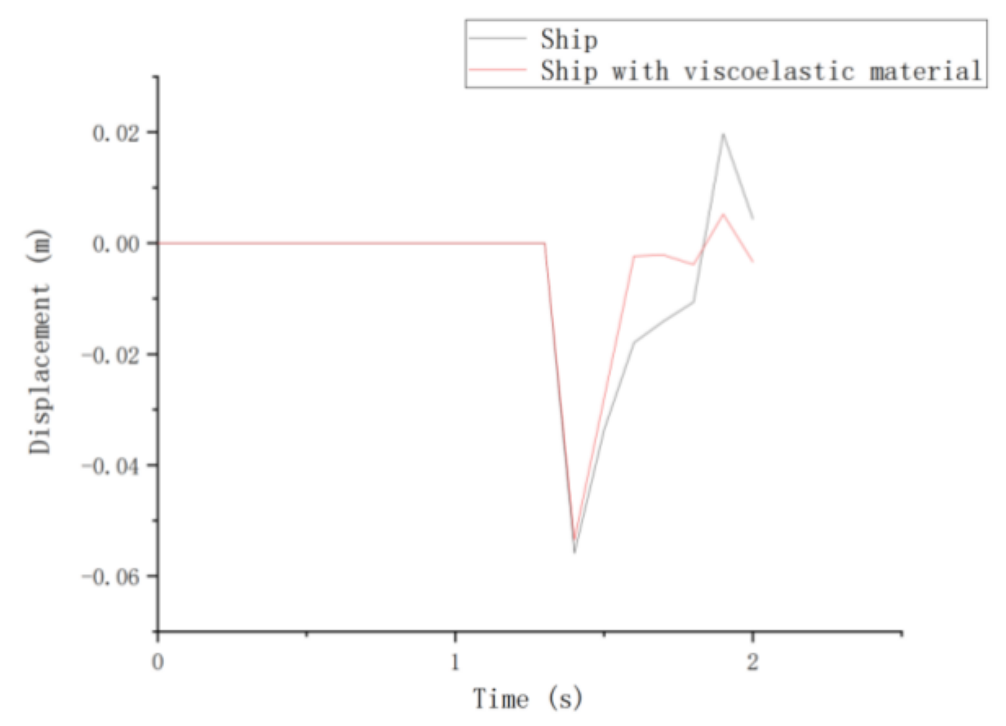

Figure 6. Displacement-time curve

\subsection{Stress comparison}

During the whole collision process, the stress curve of the collision contact point changing with time is shown in Figure 7.

For the model without viscoelastic materials, the range of the change of stress is larger, and the maximum stress is higher than that of the model with viscoelastic materials. By reducing the amount of stress applied to the ship, this material reduces the amount of strain applied to the ship, avoiding irreparable damage.

The aforementioned hysteresis of viscoelastic materials is well illustrated in Figure 7. As shown in the figure, after the collision, the fluctuation of the ship model with viscoelastic materials is significantly slower than that of the ship model without viscoelastic materials.

The hysteresis of the material is also illustrated by the twists of the viscoelastic ship at its stress peak. Differing from the other model, the ship with viscoelastic materials did not exhibit sharp stress changes before and after reaching the maximum stress. When the strain changed instantaneously, the ship did not immediately respond to the stress due to the viscoelastic material, which fully reflects the "viscosity" of the material. This feature allows ships to undertake sudden and intense stress changes, thus protecting their structure. 


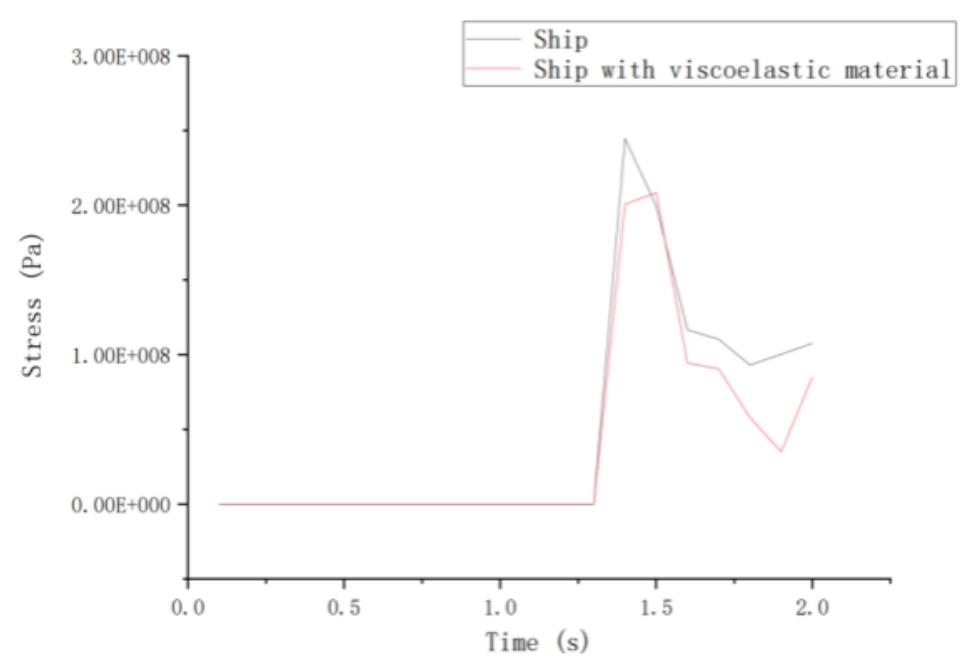

Figure 7. Stress-time curve

\section{Conclusion and prospect}

In this article, the idea of using viscoelastic materials in the side structures of ships has been proposed. The Abaqus finite element analysis software was used to analyze and compare the impact on ships with and without such materials during collisions. By modeling ships and viscoelastic materials as well as simulating collisions, several conclusions have been drawn.

(1) The hysteresis of viscoelastic materials allows the damaged part of ships to return to its initial state more quickly.

(2) Viscoelastic materials allow ships to reduce the amplitude and frequency of subsequent waves brought about by collisions.

(3) Viscoelastic materials alleviate the stress changes of ships in the process of collision.

However, compared to traditional steel structures, the cost of viscoelastic materials is higher, and the laying process is more complicated. Therefore, it is difficult to lay viscoelastic materials over the whole ship. The optimization of the laying scheme should be the focus of subsequent research.

\section{Disclosure statement}

The author declares that there is no conflict of interest.

\section{References}

[1] Kitamura Om 1998, Comparative Study on Collision Resistance of Side Structure. Marine Technology, 34(2): 293-308.

[2] Lee JW, Petershagen H, Rorup J, et al., 1999, A New Concept of Energy Absorbing System for the Double Hull Tanker. SOTECH, 3(1): 12-26.

[3] Wang Z, Gu Y, 2002, A Crashworthy Type of Double Hull Structure of VLCC. Journal of Ship Mechanics 6.1 : 27-36.

[4] Ludolphy H, 2001, Proceedings of the 2nd International Conference on Collision and Grounding of Ships, 2001: The Unsinkable Ship - Development of the Y-Shape Support Web. Copenhagen, Denmark. 
[5] Klanac A, Ehlers S, Tabri K, et al., 2005, 12th International Congress of the International Maritime Association of the Mediterranean, IMAM 2005 - Maritime Transportation and Exploitation of Ocean and Coastal Resources, September 26-30, 2005: Qualitative Design Assessment of Crashworthy Structures. Taylor and Francis/Balkema, Lisboa, Portugal, 461-469.

[6] Crupi V, Epasto G, Guglielmino E, 2013, Comparison of Aluminium Sandwiches for Lightweight Ship Structures: Honeycomb Vs. Foam. Marine Structures, 30: 74-96.

[7] Dou S, 2015, Research on Viscoelastic Damping Control Technique for Vibration and Acoustic Radiation of Shell Structure. Harbin Institute of Technology.

[8] Zheng X, 2012, 87,000 DWT Bulk Carrier Hull Structure Design Based On CSR. Shanghai Jiao Tong University. 